\title{
SISTEMA MECÁNICO DESTINADO A LA COMPENSACIÓN PASIVA DE ACELERACIONES PARA EL TRANSPORTE DE CARGAS DELICADAS EN VEHÍCULOS DE CARRETERA
}

\author{
Pedro J. Fernández-Concellón \\ Estudiante de Doctorado Ingeniería Mecánica, Universidad de Zaragoza, España \\ Santiago Baselga-Ariño \\ Profesor Titular, Universidad de Zaragoza, España
}

\section{RESUMEN}

Los sistemas pasivos para compensar aceleraciones vienen utilizándose en vehículos terrestres desde mediados del siglo XX. Su aplicación y evolución se ha dado principalmente en el transporte ferroviario de pasajeros, pero también han sido aplicados a vehículos de carretera destinados al transporte de mercancías y a otros dispositivos mecánicos empleados en la vida diaria. En esta ponencia se presenta un sistema de mecánico, alternativo a los existentes, que permite compensar de forma pasiva las aceleraciones a que se ven sometidas las cargas transportadas en el habitáculo de carga de un vehículo terrestre. Se trata de un sistema mecánico pasivo que permite que las cargas transportadas con él puedan oscilar tanto longitudinal como transversalmente, teniendo como limitaciones de su movimiento al propio habitáculo de carga, la disposición de los sus componentes y el tipo de uniones empleadas entre estos. La posibilidad de variar el tipo de uniones del sistema permite que éste adapte su comportamiento al movimiento más predominante del vehículo o a la dirección en que se desea proteger principalmente a la carga transportada. Su aplicación se ha orientado al transporte de cargas delicadas y al transporte de pacientes, dado que se ha detectado que en este último campo no existe tal aplicación y podría ser beneficioso para el estado de salud de estos. Se ha planteado y resuelto el modelo matemático de su comportamiento dinámico y existe un prototipo sobre el que se están llevando a cabo pruebas experimentales para contrastar los resultados teóricos con los reales. Su efectividad ha sido comprobada mediante simulación por ordenador y actualmente se está trabajando en la fase experimental.

\section{INTRODUCCIÓN}

\subsection{Revisión del estado de la técnica}

Los sistemas pasivos compensadores de aceleraciones comenzaron a utilizarse en vehículos guiados a principios del siglo XX. La compensación se llevaba a cabo permitiendo oscilar al vehículo lateralmente de forma natural, lo que permitía durante los pasos por curva llevar a cabo estas maniobras más rápido que con un vehículo ordinario y mejorar las condiciones de confort de los pasajeros (Toran, 1977 y 1979) y (Persson, y otros, 2009). Esto último ha sido estudiado en profundidad por numerosos investigadores dado que estas oscilaciones, 
dependiendo de factores como las frecuencias de oscilación, el tipo de movimientos, su intensidad, factores fisiológicos de las personas, etc., pueden afectar perjudicialmente a los pasajeros provocándoles mareos y malestar (Persson, y otros, 2009) y (Beard y Griffin, 2014).

Además de su aplicación en vehículos guiados, los sistemas pasivos de compensación de aceleraciones han sido utilizados en vehículos suspendidos guiados, como el monorail (Guenther y Sergeant, 2007), en vehículos de tres ruedas, formando parte de sus sistema de dirección, (Parham, 1977), (Coil, 1984) y (Barker, y otros, 2008) y de una forma más polivalente, en asientos, independientemente del vehículo en el que estos se instalen (Gribble, 2012).

En lo que respecta al transporte de cargas delicadas, ha sido utilizado para el transporte de vidrio en un semirremolque (Robertson, 1986), aislando a la carga parcialmente, permitiéndo su oscilación lateralmente, preservando su estado e integridad.

En relación al transporte de pacientes en vehículos de emergencia no se han encontrado de forma directa sistemas que permitan compensar aceleraciones de forma pasiva a través de las oscilaciones. Sin embargo, en el diseño de sistemas empleados para el transporte de pacientes podemos encontrar algunos ejemplos con similitudes con el sistema que se presenta aquí. Desde una camilla transportada manualmente que presenta unos asideros articulados en sus extremos, que le permite oscilar lateralmente (Grinberg, y otros, 2014) hasta un sistema de elevación y sujeción de la camilla robotizado, que aunque ligeramente, permite su oscilación en el interior del vehículo (Menna, 2013).

\subsection{El sistema objeto de estudio}

El sistema al que se refiere esta ponencia permite unir una plataforma o base de carga a un vehículo permitiendo un movimiento relativo de ésta respecto del vehículo. La unión puede realizarse a través de una o varias barras que presentan articulaciones en sus extremos. Dependiendo del tipo de articulaciones empleadas, el movimiento relativo de la base respecto de la barra y el de la base respecto del vehículo varía. Asimismo, las dimensiones del habitáculo donde se instale el sistema y las dimensiones de éste y de la base condicionarán el movimiento del conjunto. Algunos ejemplos, particularizados para el caso de emplear un sistema con 4 barras, pueden verse en la figura 1.
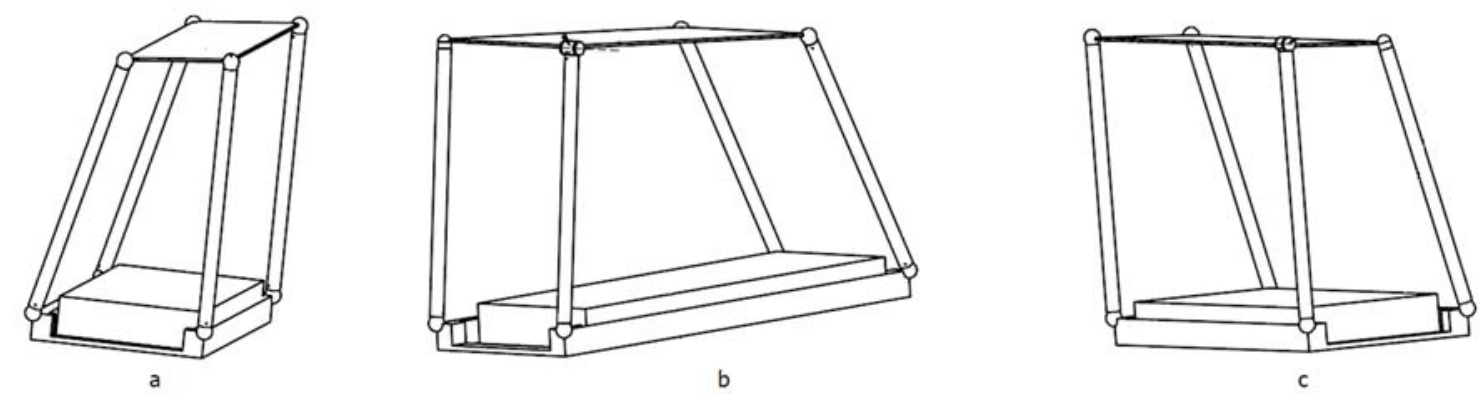

Fig. 1 - Diseños para un sistema de 4 barras

En lo que respecta al transporte de cargas delicadas, las limitaciones en el movimiento de la 
base de carga están condicionadas por los aspectos dimensionales mencionados anteriormente y por aspectos relacionados con la estabilidad del vehículo. Sin embargo, en lo que respecta al transporte de pacientes, existe una normativa de obligado cumplimiento a nivel internacional que define claramente cómo debe estar diseñado el habitáculo de carga de una ambulancia y las fijaciones de la camilla (Comité Europeo de Normalización (CEN), 2012 y 2015).

\section{METODOLOGÍA}

Una vez presentado el sistema objeto de estudio y el contexto que lo rodea, se va a detallar el análisis que se ha llevado a cabo para justificar la aplicabilidad del sistema para el propósito que ha sido diseñado y su efectividad.

Para poder comprender mejor el comportamiento dinámico del sistema se ha planteado el modelo matemático de éste, en el caso de que el sistema estuviera constituido por cuatro barras, una base de carga y cuatro amortiguadores bajo su base para dotarle de la estabilidad requerida para sus movimientos (ver figura 2 ).
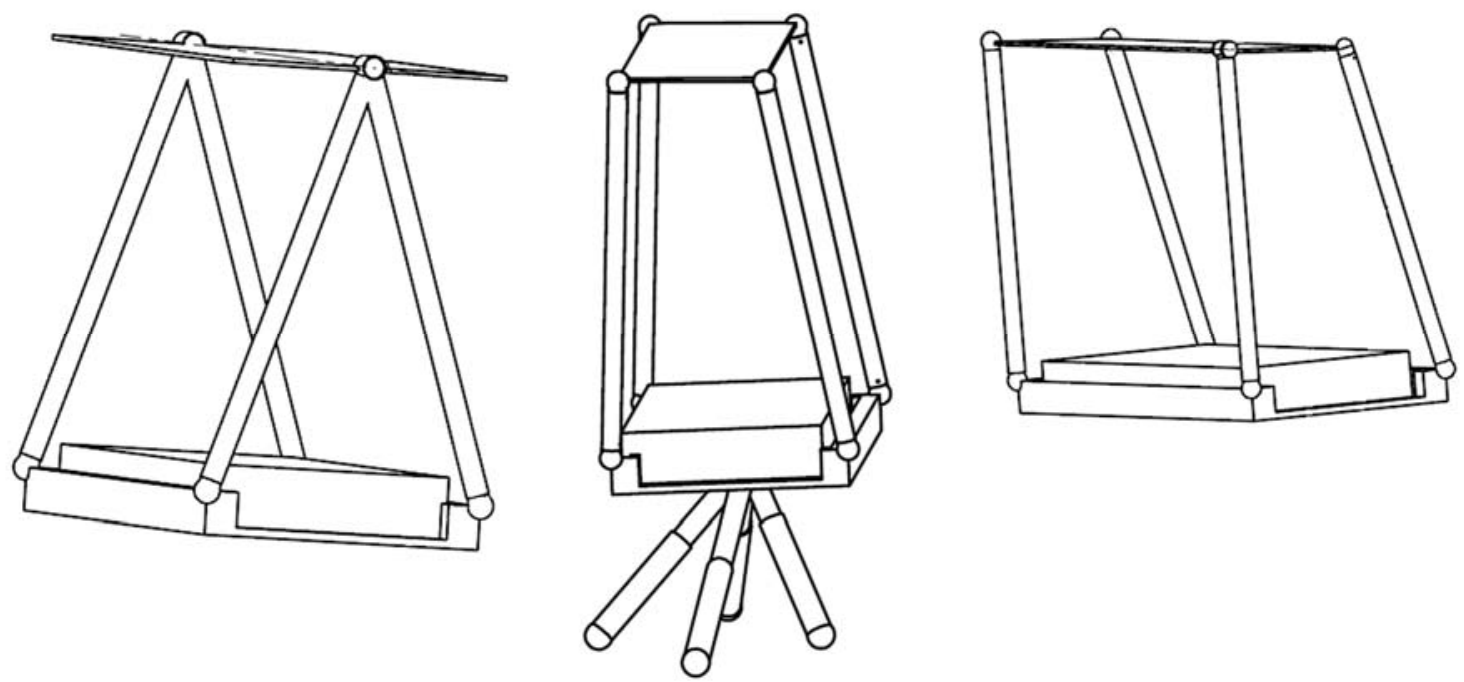

\section{Fig. 2 - Diseño analizado}

En esta configuración (tanto para las articulaciones de revolución colocadas en la dirección longitudinal como transversal, respectivamente como para las esféricas), se ha realizado un análisis cinemático a fin de encontrar las limitaciones cinemáticas que su propio diseño le confiere y un análisis vibracional, para analizar sus frecuencias naturales y la estabilidad del sistema en las condiciones de funcionamiento a que se va a ver expuesto.

Tras esto, se ha llevado a cabo una fase experimental a fin de validar tanto el modelo matemático planteado, como el diseño y los criterios de diseño adoptados como válidos en la fase teórica. 


\subsection{Modelo matemático}

El sistema planteado es un sistema de ecuaciones diferenciales algebráicas no lineales de segundo orden y su resolución se ha llevado a cabo mediante el método numérico de Newton-Raphson. Actualmente, existen métodos implementados en programas de ordenador que resuelven estos sistemas gracias al empleo de integradores optimizados y de simplificaciones que les permiten obtener soluciones precisas en un tiempo de cálculo breve. Aquí se ha resuelto el sistema sin emplear simplificaciones, a fin de comparar métodos y comprender mejor el comportamiento dinámico del sistema. El modelo matemático planteado es independiente del vehículo en el que vaya a instalarse el sistema, por lo que puede ser útil para analizar su comportamiento en cualquier situación.

\subsection{Análisis cinemático}

El análisis cinemático llevado a cabo ha permitido detectar los límites máximos de inclinación que puede tener la base de carga, dependiendo de cómo está unida al techo del vehículo, de la disposición de los amortiguadores y del espacio disponible en el habitáculo de carga.

Las uniones que se han empleado en el análisis llevado a cabo son articulaciones esféricas, tipo cardan y de revolución (bisagras). Estas articulaciones condicionan los grados de libertad del sistema y el tipo de oscilación alcanzada (longitudinal/transversal o mixta).

La disposición de las barras influye también en el movimiento del sistema. Las configuraciones más representativas del sistema analizado (figura 2) presentan movimientos asimilables al del péndulo y al del cuadrilátero articulado.

En todos los casos, se ha ubicado a los amortiguadores estabilizadores del movimiento libre del sistema justo debajo de la base de carga, evitando que los puntos de fijación de estos con el habitáculo de carga no sobresalgan del contorno de la base. Asimismo, se ha restringido la carrera de los amortiguadores para que estos alcancen unas posiciones límite tales que como mucho queden perpendiculares a la base de carga.

Teniendo en cuenta estas restricciones de diseño, para un habitáculo de carga determinado, se han determinado áreas de diseño en las que pueden seleccionarse las dimensiones de los componentes del sistema y los parámetros que definen su disposición a fin de poder inclinar la base de carga de forma que se pueda llegar a alcanzar una compensación de aceleraciones significativa.

\subsection{Análisis vibracional}

Una vez determinado cómo puede configurarse el sistema, ha sido necesario analizar cómo influye en su respuesta en frecuencias las variaciones dimensionales de los parámetros que lo configuran y la carga transportada con él. Tras este análisis, se ha estudiado cómo influye el coeficiente de amortiguamiento de los amortiguadores en la estabilidad del sistema y en la rapidez de su respuesta. Esta última parte se ha analizado simulando el movimiento del sistema como si éste recorriese un tramo de carretera en el que se encuentran separadas entre sí, dos curvas de sentidos contrarios. El trayecto, mostrado en la figura 3, es coherente con 
la reglamentación nacional en vigor, tanto por la geometría del mismo como por la velocidad del vehículo que lo recorre (Ministerio de Fomento, 2000).

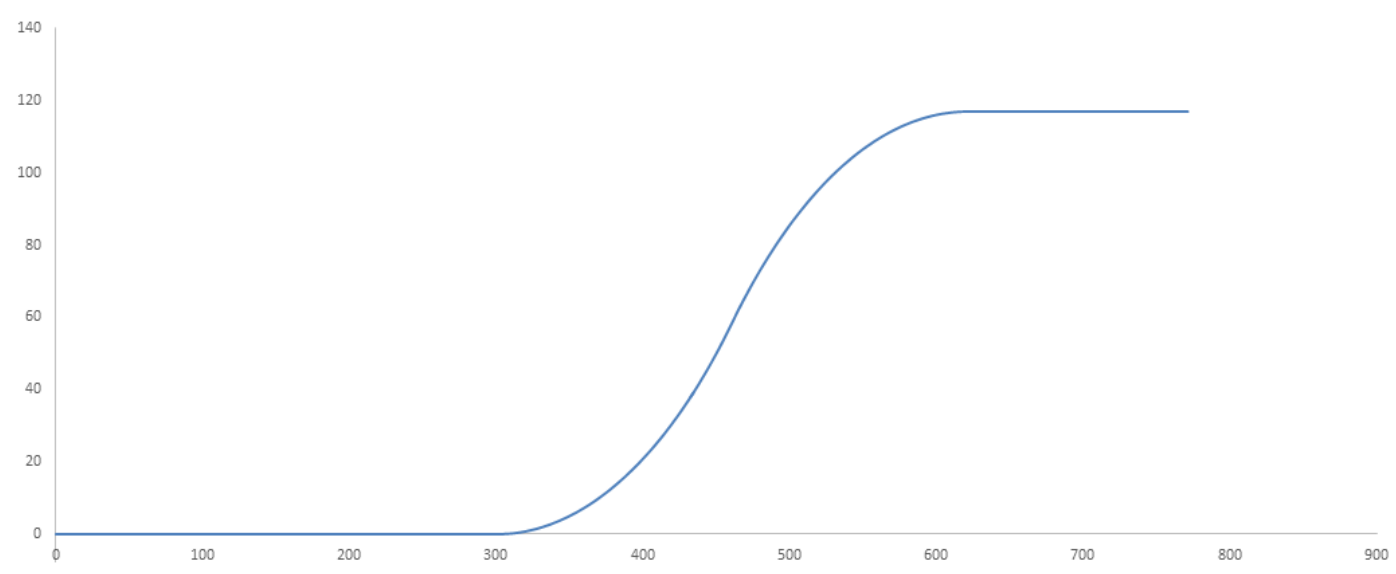

Fig. 3 - Recorrido de simulación

\section{RESULTADOS Y CONCLUSIONES}

\subsection{Analísis cinemático y dinámico}

Los resultados obtenidos en este análisis muestran que, teniendo en cuenta unas dimensiones específicas del habitáculo de carga, puede definirse un área de diseño válida para configurar el sistema con unas dimensiones tales en que la compensación pasiva de aceleraciones sea posible. Aspectos en común encontrados para diferentes habitáculos de carga son: diseños que sitúan el centro de masas del sistema próximo al suelo, al dejar poco espacio libre para el movimiento de los amortiguadores, limitan considerablemente la oscilación del sistema. De forma análoga sucede con diseños que sitúan éste muy elevado, dado que los amortiguadores quedan rápidamente perpendiculares a la base de carga. Diseños con un movimiento más parecido al del péndulo son capaces de compensar mejor las aceleraciones que los que presentan un movimiento similar al del cuadrilátero articulado, por lo que puede ser interesante combinar ambos comportamientos en función de la dirección donde se considere más importante alcanzar la compensación.

Dentro de la zona definida como apta para la compensación durante el análisis cinemático, y en función de la carga a transportar con el sistema, pueden definirse los coeficientes de amortiguamiento necesarios para los amortiguadores empleados, a fin de obtener un comportamiento estable del conjunto. El sistema debe ser capaz de estabilizar al sistema tan rápido como pueda para que sea capaz de adaptarse al movimiento del vehículo, en condiciones normales de circulación.

\subsection{Aplicación para el transporte de pacientes}

En el caso de instalar el sistema en el interior de un vehículo medicalizado, donde las dimensiones libres para el movimiento del sistema son reducidas, pueden obtenerse compensaciones de aceleraciones tanto longitudinalmente como transversalmente. 
Considerando unas dimensiones del habitáculo de carga de 2 metros de largo, 1,5 metros de ancho y 2 metros de alto y para el sistema de fijación mostrado en la figura $\mathbf{2}$, los valores de compensación de aceleraciones alcanzados se indican en la tabla $\mathbf{1}$.

\begin{tabular}{|l|c|c|c|c|}
\hline \multirow{2}{*}{ Tipo de articulaciones } & \multicolumn{2}{|c|}{ Ángulo máx. Inclinación $\left(^{\circ}\right)$} & \multicolumn{2}{|c|}{ Compensación (\%) } \\
\cline { 2 - 5 } & Lateral & Frontal & Lateral & Frontal \\
\hline Esféricas o Cardan & 3,26 & 2,9 & 18,2 & 32,1 \\
\hline Revolución & 3,26 & 2,9 & 18,2 & 32,1 \\
\hline
\end{tabular}

Tabla 1 - \% de compensación alcanzada

Los valores mostrados en la tabla anterior son los valores alcanzados durante la simulación. En otras condiciones de simulación, estos variarán. No obstante, del análisis cinemático se pueden extraer los valores límite con que la base de carga puede inclinarse. Estos, para el caso analizado, son de $5,76^{\circ}$ y $3,44^{\circ}$, en las direcciones longitudinal y lateral respectivamente.

En lo que respecta a la estabilidad en la respuesta del sistema, en la figura 4 puede apreciarse cómo el sistema, en función del coeficiente de amortiguamiento seleccionado para los amortiguadores, es capaz de responder de forma estable durante su paso por las dos curvas en zig-zag.

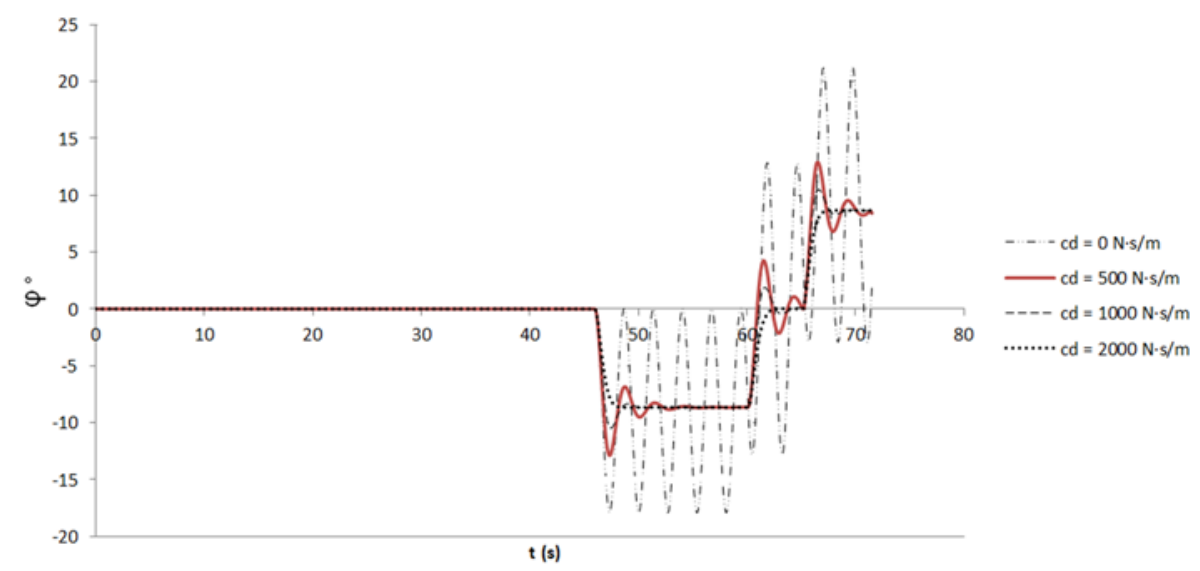

Fig. 4 - Estabilidad de la respuesta 


\section{BIBLIOGRAFÍA}

TORAN, ÁNGEL. Speed and track curvature suspension control system. US4041878 EE.UU., 08 16, 1977. A.

TORAN, ÁNGEL AND TORAN, TOMÁS. Pendular suspension system. CA1053975 Francia, 05 08, 1979. A1.

PERSSON, RICKARD, GOODALL, ROGER M. AND SASAKI, KIMIAKI. (2009) Carbody tilting - technologies and benefits Vehicle System Dynamics: International Journal of Vehicle Mechanics and Mobility, pp. 949-981

BEARD, GEORGE F. AND GRIFFIN, MICHAEL J. (2014) Motion sickness caused by roll-compensated lateral acceleration: Effects of centre-of rotation and subject demographics Journal of Rail and Rapid Transit, Vol. 228 (I), pp. 16-24.

GUENTHER, KARL AND SERGEANT, WILFRED P.E. Overhead Suspended Transportation System and Method. US20070017410 EE.UU., 01 25, 2007. A1

PARHAM, HAROLD D. Three-wheel motorcycle. US4064957 EE.UU., 12 27, 1977. A.

COIL, STANLEY F. Tiltable three-wheeled vehicle. US4469344 EE.UU., 09 04, 1984. A

BARKER, M., ET AL., (2008) Steady-state steering of a tilting three-wheeled vehicle Vehicle System Dynamics, Vol. 48, pp. 815-830. 1744-5159

GRIBBLE, JAMES E. Self-leveling, gravity-stabilized, sliding and tilting support for a chair. US20120267503 EE.UU., 10 25, 2012. A1.

ROBERTSON, PAUL E. Trailer structure for transporting sheet glass and frangible material. US4626017 EE.UU., 12 02, 1986. A.

GRINBERG, JOSHUA, ET AL. Weight-stabilizing stretcher. US20140007350 EE.UU., 01 09, 2014. A1.

MENNA, EZIO. Ambulance vehicle for transport of patients, injured persons and the like. US20130127198 EE.UU., 05 23, 2013. A1.

COMITÉ EUROPEO DE NORMALIZACIÓN (CEN). Equipos para el transporte de pacientes utilizados en ambulancias de carretera. Parte 5: Soporte de la camilla [trans.] AENOR. Madrid: AENOR, Noviembre 2012. UNE-EN 1865-5. M 39136:2012

COMITÉ EUROPEO DE NORMALIZACIÓN (CEN). Vehículos de transporte sanitario y 
sus equipos. Ambulancias de carretera. [trans.] AENOR. Madrid: AENOR, Febrero 2015. UNE-EN 1789:2007+A2. M 6535:2015

MINISTERIO DE FOMENTO. Orden de 27 de diciembre de 1999 por la que se aprueba la Norma 3.1-IC. Trazado, de la Instrucción de Carreteras. Boletín Oficial del Estado. [Boletín Oficial del Estado]. Madrid, España: Ministerio de Fomento, febrero 2, 2000. 28. 28. 